\title{
Evidence for dark matter on different scales from the KLUN galaxy sample
}

\author{
M. O. Hanski, P. Teerikorpi and T. Ekholm
}

Tuorla Observatory, Piikkiö, Finland

G. Theureau

Osservatorio Astronomico di Capodimonte, Naples, Italy

Yu. Baryshev

Astronomical Institute of St.Petersburg University, St.Petersburg, Russia

G. Paturel and P. Lanoix

Observatoire de Lyon, Lyon, France

\section{Introduction}

The KLUN (Kinematics of the Local UNiverse) sample of 6600 spiral galaxies is used in studying dark matter on different scales:

- The Morphological Type dependence of the zero-point of the Tully-Fisher relation indicates mass to light ratio $M / L \approx 9-16$ on galactic scales.

- Preliminary results from a study of selection effects influencing double galaxies give a larger value $M / L \approx 30-50$.

- Study of the Perseus-Pisces supercluster, using Malmquist bias corrected TF distances and Tolman-Bondi solutions indicates $M / L \approx 200-600$ for large clusters. Similar results were obtained in our previous work on Virgo galaxies.

- Application of a modified version of Sandage-Tammann-Hardy test of the linearity of Hubble law inside the observed hierarchical (fractal) galaxy distribution up to $200 \mathrm{Mpc}$ suggests that either $\Omega_{0}$ is very small $(0.01)$ or the major part of the matter is uniformly distributed dark matter.

\section{KLUN}

The KLUN sample currently contains 6,600 spiral galaxies having measurements of isophotal diameter $\left(D_{25}\right), \mathrm{HI}$ line width, radial velocity, and $B$-magnitude. The sample is selected according to apparent diameter and is complete down to $D_{25}=1.6$ arcmin. Morphological type ranges from Sa to $\mathrm{Sdm}(T=1-8)$. The 
data are extracted from the homogenized compilation catalogue LEDA (LyonMeudon Extragalactic database, http://www-obs.univ-lyon1.fr/leda) and complemented with redshift and H I spectrum measurements. Previously the KLUN sample has been used for the determination of the Hubble parameter using the Tully-Fisher (TF) relation. After taking properly into account the Malmquist selection and calibrator sample biases, and using the type dependent TF relation, we obtained $H_{0} \approx 55 \mathrm{~km} \mathrm{~s}^{-1} \mathrm{Mpc}^{-1}$ with both the direct (Theureau et al. 1997b) and the inverse (Ekholm et al. 1999) TF relation.

\section{Type dependence in the TF relation and disk + bulge + dark halo model}

The revealed Hubble type dependence in the zero-point of the TF relation has been interpreted with a simple mass model (exponential disk + bulge + dark halo $\propto$ luminous mass (Theureau et al. 1997a)). The fraction of dark mass was found to be in the range $50-80 \%$ within the radius that the TF measurements refer to. This corresponds to $M / L \approx 9-16$, depending on the type. A better evaluation is expected when one takes into account a luminosity dependent $M / L$ ratio (Hanski \& Teerikorpi, in prep.).

Table 1. The simple model described in Theureau et al. (1997a) uses a common $M / L=3.72$ for the bulge, and type dependent $M / L$ value for the disk component of spirals. Adding the gas masses and assuming constant dark mass fraction the total $M / L$ of spirals can be estimated by fitting the observed TF zero points to the ones predicted by this model. The values below are from Table 3 . in Theureau et al. (1997a).

\begin{tabular}{ccccc}
$T^{a}$ & $\left(M / L_{B}\right)_{\text {disk }^{b}}{ }^{b}$ & $y_{\mathrm{HI}}{ }^{c}$ & $y_{\text {tot }}{ }^{d}$ & $M / L\left(g r_{0}\right)^{e}$ \\
\hline 1 & 1.44 & 0.03 & 0.05 & 15.8 \\
2 & 1.37 & 0.04 & 0.06 & 15.2 \\
3 & 1.08 & 0.06 & 0.08 & 14.3 \\
4 & 0.96 & 0.07 & 0.09 & 12.7 \\
5 & 0.87 & 0.09 & 0.11 & 12.6 \\
6 & 0.64 & 0.10 & 0.12 & 9.9 \\
7 & 0.64 & 0.12 & 0.12 & 8.8 \\
8 & 0.76 & 0.14 & 0.14 & 13.2 \\
\hline
\end{tabular}

${ }^{a}$ morphological type

${ }^{b}$ disc mass-to-light ratio

${ }^{c, d} \mathrm{Hr}$ and total gas masses

${ }^{e}$ mass-to-luminosity ratio inside $\mathrm{TF}$ measuring radius

Such values are somewhat larger than what is obtained from the double galaxy sample by Karachentsev, and work is in progress to identify the reason for the inconsistency (Teerikorpi, in prep.). Preliminary results, taking into account 
the incompleteness in large separation distances, suggest that Karachentsev's sample gives evidence for $M / L \approx 30$ for spiral-spiral pairs.

\section{Masses of the Virgo and Perseus-Pisces superclusters and the Tolman-Bondi model}

We have studied the Perseus-Pisces (PP) supercluster (Hanski et al., 1999) using Malmquist-bias corrected TF distances and the Tolman-Bondi solutions. Virial masses indicate $M / L=200-600$ for the largest PP clusters (Fig. 1). Using matter distribution with an excess density $\propto r^{-2}$, where $r$ is distance from PP, and a void between PP and the Local Group, we get a good fit between the Tolman-Bondi model and KLUN data points. If the $M / L$ values are valid elsewhere in the Universe, we obtain $\Omega_{0}=0.1-0.3$. The difference between zero and non-zero cosmological constant was found to be negligible in the TolmanBondi calculations.

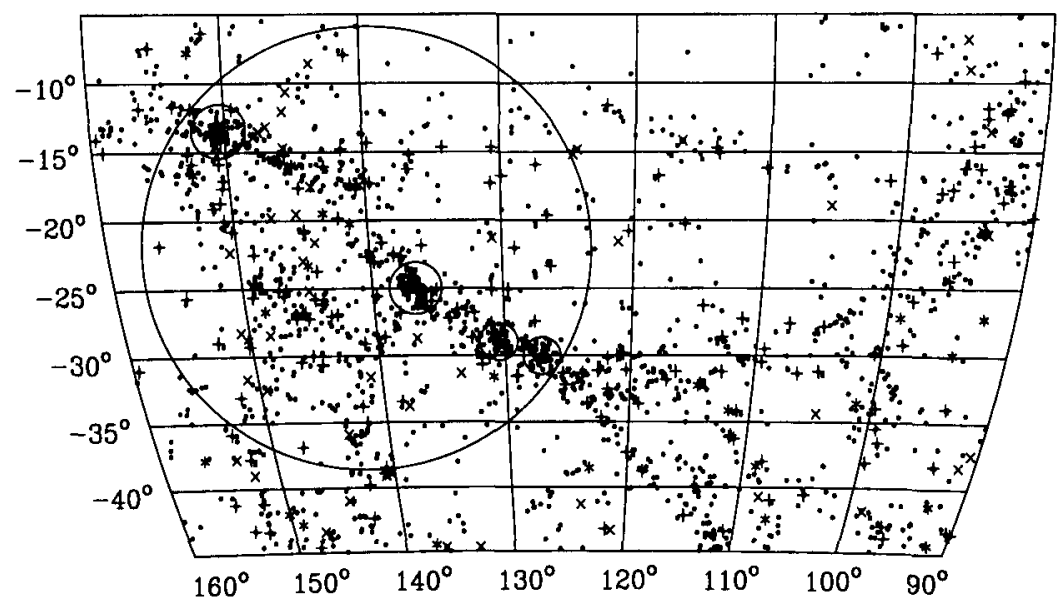

Figure 1. LEDA (o) and KLUN galaxies $(+, *$ and $\times)$ in the Perseus-Pisces region. The large circle is the $15 h^{-1} \mathrm{Mpc}$ sphere at $\left(140.2^{\circ},-22.0^{\circ}\right)$ containing the main concentration. Small circles are the four densest clusters, Perseus, A262, 0122+3305 and Pisces, from upper left to lower right. Using virial and other estimators by Heisler et al. (1985) we calculated $M / L$ ratios of $280-540,200-390,250-550$, and 260-590 for these four clusters. See Hanski et al. (1999) for details.

The applicability of the Tolman-Bondi solution even in regions where the luminous matter distribution is not spherically symmetric has been demonstrated in our previous and new work on the Virgo supercluster (Teerikorpi et al. 1992, Ekholm et al., 1999). This suggests that the dark matter may be more symmetrically distributed. 


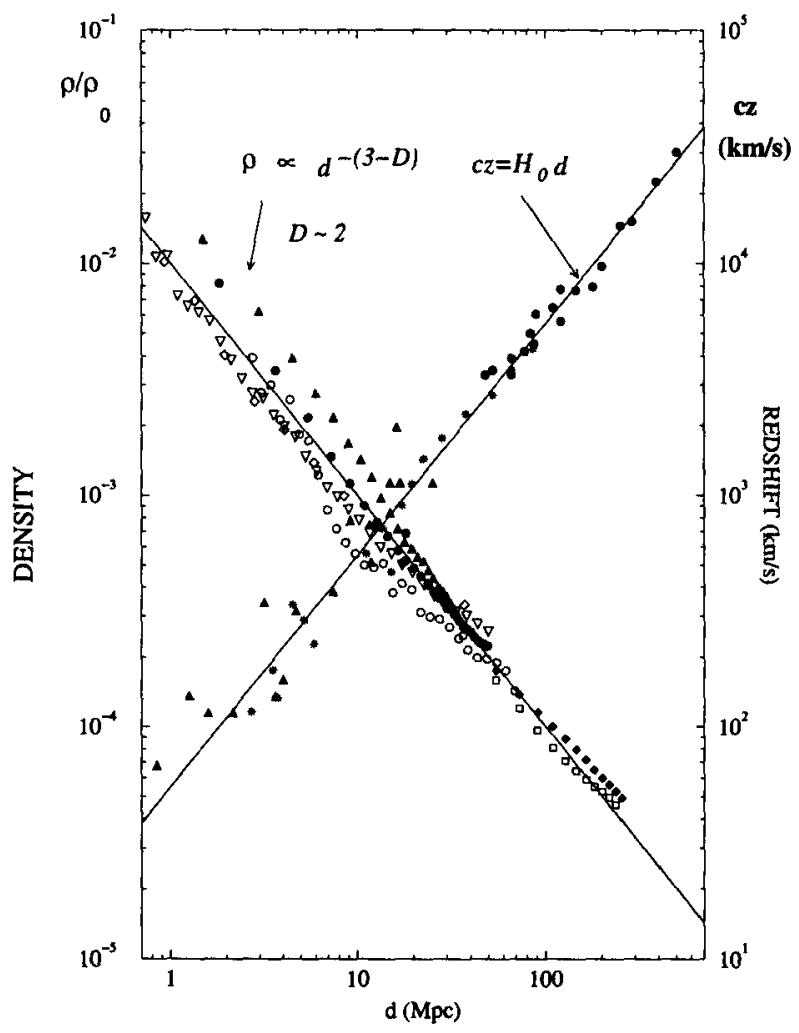

Figure 2. The Hubble law vs. the galaxy distribution.

\section{Linearity of the Hubble law inside a fractal galaxy distribution}

We have applied a modified version of the Sandage-Tammann-Hardy test of the linearity of the Hubble law inside a hierarchical (fractal) galaxy distribution using a linear perturbation approximation for the velocity-distance law (Baryshev et al., 1998).

If fractality with dimension $D \approx 2$ extends up to $200 \mathrm{Mpc}$, as suggested by certain studies, including our own using KLUN (Teerikorpi et al., 1998), a small $\Omega_{0}(\leq 0.01)$ is required to produce a good Hubble law. A large $\Omega_{0}$ is possible if the dark matter uniformly fills space. E.g. with $\Omega_{0}=1$, one derives $\Omega_{\text {dark }}=0.99$, and the fractality is restricted to luminous matter.

\section{References}

Baryshev, Yu., Sylos Labini, F., Montuori, M., Pietronero, L., \& Teerikorpi, P. 1998, Fractals, Vol. 6, No. 3, 231

Ekholm, T., Teerikorpi, P., Theureau, G. et al. 1999, A\&A, 347, 99 
Ekholm, T., Lanoix, P., Paturel, G., \& Teerikorpi, P., 1999, A\&A, submitted Hanski, M., Theureau, G., Ekholm, T., \& Teerikorpi, P. 1999, A\&A, submitted Heisler, J., Tremaine, S., \& Bahcall, J. 1985, ApJ, 298, 8

Teerikorpi, P., Bottinelli, L., Gouguenheim, L., Paturel, G. 1992, A\&A260, 17 Teerikorpi, P., Hanski, M., Theureau, G. et al. 1998, A\&A334, 395

Theureau, G., Hanski, M., Teerikorpi, P. et al. 1997a, A\&A, 319, 435

Theureau, G., Hanski, M., Ekholm, T. et al. 1997b, A\&A, 322, 730 